\title{
Well-Posedness for the 2D Non-Autonomous Incompressible Fluid Flow in Lipschitz-like Domain
}

\author{
YANG Xin-Guang ${ }^{1}$ and WANG Shubin ${ }^{2 *}$ \\ ${ }^{1}$ Department of Mathematics and Information Science, Henan Normal University, \\ Xinxiang 453007, China. \\ 2 School of Mathematics and Statistics, Zhengzhou University, Zhengzhou 450001, \\ China.
}

Received 28 September 2018; Accepted 16 March 2019

\begin{abstract}
This paper is concerned with the global well-posedness and regularity of weak solutions for the 2D non-autonomous incompressible Navier-Stokes equation with a inhomogeneous boundary condition in Lipschitz-like domain. Using the estimate for governing steady state equation and Hardy's inequality, the existence and regularity of global unique weak solution can be proved. Moreover, these results also hold for 2D Navier-Stokes equation with Rayleigh's friction and Navier-Stokes-Voigt flow, but invalid for three dimension.
\end{abstract}

AMS Subject Classifications: 35B40, 35B41, 35Q30, 76D03, 76D05

Chinese Library Classifications: $\mathrm{O} 175.27$

Key Words: Non-autonomous Navier-Stokes equation; Lipshitz-like domain; background flow function.

\section{Introduction}

The incompressible Navier-Stokes equation is a well-known hydrodynamical model which plays a important role in understanding continuous medium mechanics. Our objective in this paper is to study the global well-posedness and its regularity for a $2 \mathrm{D}$ incompressible non-autonomous fluid flow with non-homogeneous boundary condition in a

*Corresponding author. Email addresses: yangxinguang@hotmail.com (X. G. Yang), wangshubin@zzu.edu.cn (S. B. Wang) 
Lipschitz-like domain:

$$
\begin{cases}\frac{\partial u}{\partial t}-v \Delta u+(u \cdot \nabla) u+\nabla p=f(t, x), & x \in \Omega, t \geq \tau \\ \operatorname{div} u=0, & x \in \Omega, t \geq \tau \\ u=\varphi, \quad \varphi \cdot n=0, & x \in \partial \Omega, t \geq \tau \\ u(\tau, x)=u_{\tau}(x), & x \in \Omega,\end{cases}
$$

wxhere $\Omega \subset \mathbb{R}^{2}$ is a bounded set which is said to be a Lipschitz-like domain if its boundary $\partial \Omega$ can be covered by finitely many balls $B_{i}=B\left(Q_{i}, r_{0}\right)$ centered at the point $Q_{i} \in \partial \Omega$ such that for each ball $B_{i}$, there exists a rectangular coordinate system and a Lipschitz function $\Psi: \mathbb{R}^{d-1} \rightarrow \mathbb{R}$ with

$$
B\left(Q_{i}, 3 r_{0}\right) \cap \Omega=\left\{\left(x_{1}, x_{2}, \cdots, x_{d}\right) \mid x_{d}>\Psi_{i}\left(x_{1}, x_{2}, \cdots, x_{d-1}\right)\right\} \cap \Omega,
$$

$\tau \in \mathbb{R}$ is an initial time. The variables $u$ represents the fluid velocity field, $p$ denotes the pressure, and $v$ is the kinematic viscosity. In addition, $n$ represents the exterior unit normal vector to $\partial \Omega, \varphi=\varphi(x)$ is a prescribed tangential boundary velocity, and $f(t, x)$ is a time-dependent forcing term.

The 2D incompressible Navier-Stokes equations with homogeneous Dirichlet or periodic boundary in smooth domain, we can refer to literature [3-7,11]. And the 2D autonomous system with non-homogenous boundary on smooth domain can be founded in $[8,9]$. For this problem extended to non-smooth, by an appropriate background flow, [1] investigated the well-posedness in less regular space and its dynamics. In this paper, we want to investigate the non-autonomous case. Firstly, we introduce the background function $\psi$, which is the solution to following problem that shares the same boundary condition $\varphi$ as (1.1):

$$
\begin{cases}\operatorname{div} \psi=0, & \text { in } \Omega \\ \psi=\varphi, \quad \varphi \cdot n=0 & \text { on } \partial \Omega\end{cases}
$$

and $\psi$ is a solution for the Stokes problem

$$
\left\{\begin{array}{l}
-\Delta \hat{u}+\nabla q=0, \quad \text { in } \Omega, \\
\operatorname{div} \hat{u}=0, \quad \text { in } \Omega, \\
\hat{u}=\varphi, \text { a.e. on } \partial \Omega \text { in the sense of nontangential convergence. }
\end{array}\right.
$$

The idea comes from [8] and [9], then [1] extended to non-smooth domains of 2D NavierStokes equation by critically invoking estimates of the Stokes problem. Then, writing 
$v=u-\psi$, problem (1.1) becomes its equivalent form:

$$
\left\{\begin{array}{lc}
\frac{\partial v}{\partial t}-v \Delta v+(v \cdot \nabla) v+(v \cdot \nabla) \psi+(\psi \cdot \nabla) v+\nabla p \\
\operatorname{div} v=0, & x \in \Omega, t \geq \tau, \\
v=0, & x \in \partial \Omega, \\
v(\tau, x)=v_{\tau}(x), & x \in \Omega,
\end{array}\right.
$$

which is a homogeneous problem. If $v$ is a solution of problem (1.4) with initial data $v_{\tau}=u_{\tau}-\psi$, then $u=\psi+v$ is a solution of problem (1.1) with initial data $u_{\tau}$. The Hadamard well-posedness of problem based on stationary system (see $[2,10])$ and the existence of a global attractor of finite fractal dimension of problem (1.4) were proved in [1], with respect to weak solutions and $f=f(x)$.

In this presented work, we consider the global well-posedness for problem (1.4) which has the features and properties:

(1) Estimates of $\psi$ on a Lipschitz-like domain established in [1] have to be critically invoked, which is necessary for well-posedness of our non-autonomous problem.

(2) The Lipschitz-like boundary requires more delicate estimates which is dependent on $\Omega$.

(3) For the non-autonomous case, we need some new inequalities to deal the external force.

(4) The regularity of global weak solution need some new delicate estimate for the fractional power operator.

The paper is arranged as: some inequalities and main results has been show in Section 2, which has the proof in Section 3.

\section{Main results}

\subsection{Preliminaries}

In this paper, we denote $E:=\left\{u \mid u \in\left(C_{0}^{\infty}(\Omega)\right)^{2}\right.$, divu $\left.=0\right\}, H$ is the closure of $E$ in $\left(L^{2}(\Omega)\right)^{2}$, $(\cdot, \cdot)$ and $|\cdot|$ denote the inner product and norm in $H$ respectively, i.e.,

$$
(u, v)=\sum_{j=1}^{2} \int_{\Omega} u_{j}(x) v_{j}(x) \mathrm{d} x, \quad \forall u, v \in\left(L^{2}(\Omega)\right)^{2} \text { and }|u|^{2}=(u, u) .
$$

$V$ is the closure of $E$ in $\left(H^{1}(\Omega)\right)^{2}$ topology, $((\cdot, \cdot))$ and $\|\cdot\|$ denote the inner product and norm in $V$ respectively, i.e.,

$$
((u, v))=\sum_{i, j=1}^{2} \int_{\Omega} \frac{\partial u_{j}}{\partial x_{i}} \frac{\partial v_{j}}{\partial x_{i}} \mathrm{~d} x, \quad \forall u, v \in\left(H_{0}^{1}(\Omega)\right)^{2} \text { and }\|u\|^{2}=((u, u)) .
$$


$H^{\prime}$ and $V^{\prime}$ are dual spaces of $H$ and $V$ respectively, where the injections $V \hookrightarrow H \equiv H^{\prime} \hookrightarrow V^{\prime}$ are dense and continuous. The norm $\|\cdot\|_{*}$ and $\langle\cdot\rangle$ denote the norm in $V^{\prime}$ and the dual product between $V$ and $V^{\prime}$ respectively.

Let $P$ be the Helmholz-Leray orthogonal projection operator from $\left(L^{2}(\Omega)\right)^{3}$ onto $H$. We define $A:=-P \Delta$ to be the Stokes operator with domain $D(A)=\left(H^{2}(\Omega)\right)^{3} \cap\left(H_{0}^{1}(\Omega)\right)^{3}$, then the operator $A: V \rightarrow V^{\prime}$ has the property $\langle A u, v\rangle=((u, v))$ for all $u, v \in V$ which is an isomorphism from $V$ into $V^{\prime} .\left\{\lambda_{j}\right\}_{j=1}^{\infty}\left(0<\lambda_{1} \leq \lambda_{2} \leq \cdots\right)$ are eigenvalues of operator $A$ for the eigenvalue problem $A u=\lambda u,\left.u\right|_{\partial \Omega}=0$ in $L^{2}(\Omega) .\left\{\omega_{j}\right\}_{j=1}^{\infty}$ is an orthonormal basis of operator $A$ corresponding to $\left\{\lambda_{j}\right\}_{j=1}^{\infty}$, i.e., $A \omega_{j}=\lambda_{j} \omega_{j}$.

We define the bilinear and trilinear operators as (see [11])

$$
\begin{aligned}
& B(u, v):=P((u \cdot \nabla) v), \quad \forall u, v \in E, \\
& b(u, v, w)=(B(u, v), w)=\sum_{i, j=1}^{2} \int_{\Omega} u_{i} \frac{\partial v_{j}}{\partial x_{i}} w_{j} \mathrm{~d} x
\end{aligned}
$$

respectively. The operators $B(u, v)$ and $b(u, v, w)$ satisfies

$$
\begin{cases}b(u, v, v)=0, & \forall u \in V, v \in\left(H_{0}^{1}(\Omega)\right)^{2}, \\ b(u, v, w)=-b(u, w, v), & \forall u, v, w \in V, \\ |b(u, v, w)| \leq C|u|^{\frac{1}{2}}\|u\|^{\frac{1}{2}}\|v\||w|^{\frac{1}{2}}\|w\|^{\frac{1}{2}}, & \forall u, v, w \in V .\end{cases}
$$

We also present Hardy's inequality

$$
\int_{\Omega} \frac{|u(x)|^{2}}{[\operatorname{dist}(x, \partial \Omega)]^{2}} \mathrm{~d} x \leq C_{3} \int_{\Omega}|\nabla u(x)|^{2} \mathrm{~d} x, \quad \forall u \in V
$$

which will be used in sequel.

\subsection{Some useful spaces}

We define the fractal operator $A^{s}(s \in \mathbb{R})$ as

$$
\begin{aligned}
& A^{s} f=\sum_{j} \lambda_{j}^{s}\left(f, \omega_{j}\right) \omega_{j}, \quad s \in \mathbb{R}, j \in \mathbb{R}, \\
& V^{s}=D\left(A^{s}\right)=\left\{g \in H: A^{s} g \in H, \sum_{i=1}^{+\infty} \lambda_{i}^{2 \alpha}\left|\left(u, \omega_{i}\right)\right|^{2}<+\infty\right\}, \\
& \left\|A^{\sigma} u\right\|=\left(\sum_{i=1}^{+\infty} \lambda_{i}^{2 \sigma}\left|\left(u, \omega_{i}\right)\right|^{2}\right)^{1 / 2} .
\end{aligned}
$$

Here $D\left(A^{s}\right)$ denotes the domain of $A^{s}$ with the inner product and the norm $\|\cdot\|_{s}$ as

$$
(u, v)_{V^{s}}=\left(A^{\frac{s}{2}} u, A^{\frac{s}{2}} v\right), \quad\|u\|_{V^{s}}^{2}=(u, u)_{V_{s}}
$$


and $V^{\frac{s+1}{2}}=D\left(A^{\frac{s+1}{2}}\right)$ with the norm $\|\cdot\|_{V^{\frac{s+1}{2}}}$. Especially,

$$
V=V^{1}, \quad V^{2}=W:=\left(H^{2}(\Omega)\right)^{3} \bigcap\left(H_{0}^{1}(\Omega)\right)^{3} .
$$

Moreover, $A^{s}$ satisfies (see [1])

$$
\|u\|_{L^{4}} \leq C_{1}\left|A^{\frac{1}{4}} u\right|, \quad \forall u \in D\left(A^{\frac{1}{4}}\right) .
$$

The Gagliardo-Nirenberg interpolation inequality

$$
\left|A^{1 / 2} u\right|^{2} \leq C_{2}\left|A^{1 / 4} u\right|\left|A^{3 / 4} u\right|, \quad \forall u \in D\left(A^{3 / 4}\right) .
$$

\subsection{Estimate of background function for (1.3)}

The background function for the Stokes problem in Lipschitz-like domain satisfies the following estimate:

(1) The background function $\psi$ which is a solution of Stokes problem satisfies the following estimates in a Lipschitz-like domain

$$
\begin{aligned}
& \sup _{x \in \Omega}|\psi(x)|+\sup _{x \in \Omega}|\nabla \psi(x)| \operatorname{dist}(x, \partial \Omega) \leq C_{4}\|\varphi\|_{L^{\infty}(\partial \Omega),} \\
& \left\||\nabla \psi| \operatorname{dist}(\cdot, \partial \Omega)^{1-\frac{1}{p}}\right\|_{L^{p}(\Omega)} \leq C_{5}\|\varphi\|_{L^{p}(\partial \Omega),} \quad 2 \leq p \leq \infty, \\
& \|\psi\|_{L^{\infty}(\Omega)} \leq C_{4}\|\varphi\|_{L^{\infty}(\partial \Omega)} .
\end{aligned}
$$

(2) If $\psi$ satisfies the problem

$$
\left\{\begin{array}{l}
\operatorname{div} \psi=0, x \in \Omega, \\
\psi=\hat{u}, \text { if } x \in\left\{x \in \Omega ; \operatorname{dist}(x, \partial \Omega)<C_{1}^{\prime} \varepsilon\right\} \\
\psi=\varphi, \text { on } \partial \Omega \text { in sense of nontangential convergence. }
\end{array}\right.
$$

Then we have

$$
\Delta \psi=\nabla\left(q \eta_{\varepsilon}\right)+F,
$$

where

$$
\begin{aligned}
& \text { Supp } \psi \subset\left\{x \in \bar{\Omega} ; \operatorname{dist}(x, \partial \Omega)<C_{2}^{\prime} \varepsilon\right\}, \\
& \|F\|_{L^{2}(\Omega)} \leq \frac{C}{\varepsilon^{\frac{3}{2}}}\|\varphi\|_{L^{2}(\partial \Omega)}, \nabla q=\triangle \hat{u}, \\
& F=0, \quad x \in\left\{x \mid \operatorname{dist}(x, \partial \Omega)<C_{1}^{\prime} \varepsilon \text { or } \operatorname{dist}(x, \partial \Omega)>C_{2}^{\prime} \varepsilon\right\},
\end{aligned}
$$

here $\varepsilon$ is an arbitrary fixed positive constant. 


\subsection{The equivalent problem}

Based on the above notations, since the Stokes operator is compact, then (1.4) is equivalent to the following homogeneous boundary value problem

$$
\left\{\begin{array}{l}
v_{t}-v \Delta v+(v(t) \cdot \nabla) v+(v(t) \cdot \nabla) \psi+(\psi \cdot \nabla) v+\nabla\left(p-v q \eta_{\varepsilon}\right)=\bar{f}-(\psi \cdot \nabla) \psi, \\
\operatorname{div} v=0, \\
\left.v\right|_{\partial \Omega}=0, \\
v(\tau, x)=v_{\tau}(x)=u_{\tau}(x)+\psi(x),
\end{array}\right.
$$

where $\bar{f}=f(x, t)+v F(x)$.

Let $v_{\tau} \in H$ or $V$, applying the Leray projector $P$ to problem (2.19), using the divergence free condition, we derive the following equivalent abstract weak form

$$
\left\{\begin{array}{l}
v_{t}+v A v+B(v, v)+B(v, \psi)+B(\psi, v)=P \bar{f}-B(\psi, \psi), \\
\operatorname{div} v=0
\end{array}\right.
$$

\subsection{Existence of global weak solution}

Let $\psi$ be a background flow function which satisfies the Stokes problem (1.3) and $v(t, x)=$ $u(t, x)-\psi(x)$, and its global weak solution can be defined as following.

Definition 2.1. Let $\Omega$ be a Lipschitz-like domain, $u_{\tau} \in H, f(x, t) \in L_{\text {loc }}^{2}\left(\mathbb{R} ; V^{\prime}\right), \varphi \in L^{\infty}(\partial \Omega)$ and $\varphi \cdot n=0$ on $\partial \Omega, u$ is called a weak solution of problem (1.1) provided that

(i) $u \in C([\tau, T] ; H), u(\cdot, \tau)=u_{\tau}$, and $\mathrm{d} u / \mathrm{d} t \in L^{2}\left(\tau, T ; V^{\prime}\right)$;

(ii) for all $v \in C_{0}^{\infty}(\Omega)$ with div $v=0$, we get

$$
\frac{\mathrm{d}}{\mathrm{d} t}<u, v>-v<u, \Delta v>-\int_{\Omega_{i, j=1}} \sum_{i} u^{i} u^{j} \frac{\partial v^{i}}{x_{j}} \mathrm{~d} x=<f, v>
$$

in the distributional sense on $[\tau, T]$;

(iii) there exists functions $\psi \in C^{2}(\Omega) \cap L^{\infty}(\partial \Omega), q \in C^{1}(\Omega)$ and $g \in L^{2}(\Omega)$ such that

$$
\begin{cases}\triangle \psi=\nabla q+\hat{g}, & \text { in } \Omega, \\ \operatorname{div} \psi=0, & \text { in } \Omega, \\ \psi=\varphi, & \text { on } \partial \Omega,\end{cases}
$$

where $\psi$ can reach its boundary values in the sense of non-tangential convergence and $u-$ $\psi \in L^{2}(\tau, T ; V)$.

The existence of global weak solutions of problem (2.20) (which is equivalent to (2.19)) can be stated as the following theorem. 
Theorem 2.1. Let $v_{\tau}(x) \in H, f(x, t) \in L_{\text {loc }}^{2}\left(\mathbb{R} ; V^{\prime}\right)$, then there exists a unique solution $v(t, x)$ of non-autonomous problem (2.20) satisfying

$$
v(t, x) \in L^{\infty}(\tau, T ; H) \bigcap L^{2}(\tau, T ; V),
$$

and $\mathrm{d} v / \mathrm{d} t$ is uniformly bounded in $L^{2}\left(\tau, T ; V^{\prime}\right)$.

Proof. Applying the Galerkin approximated method and compact argument as in [1] to our non-autonomous problem, this result could be established, see Section 3.1.

The continuous dependence of global solutions on initial data will be presented in following theorem.

Theorem 2.2. Let $u_{\tau} \in H, f \in L_{\text {loc }}^{2}\left(\mathbb{R} ; V^{\prime}\right), \varphi \in L^{\infty}(\partial \Omega)$, and $\varphi \cdot n=0$ on $\partial \Omega$. Then, we obtain that the weak solution satisfies $u(t, x) \in L^{4}([\tau, T] \times \Omega)$.

Moreover, the problem (1.1) possesses a unique weak solution $u(t, x) \in L^{\infty}(\tau, T ; H) \cap L^{2}(\tau, T ; V)$ which is continuously dependent on the initial data, i.e., $u(t, x) \in C(\tau, T ; H)$.

Proof. See Section 3.1.

Remark 2.1. For the above theorem, if we consider the inhomogeneous boundary condition for 2D incompressible Navier-Stokes equation with Rayleigh's friction (See [13]) in Lipschitz-like domain

$$
\left\{\begin{array}{l}
\frac{\partial u}{\partial t}-v \Delta u+(u \cdot \nabla) u+\alpha u+\nabla p=f(t, x) \\
\operatorname{div} u=0
\end{array}\right.
$$

then the system (2.21) possesses a global weak solution $u(t, x)$ satisfying

$$
u(t, x) \in L^{\infty}(\tau, T ; H) \cap L^{2}(\tau, T ; V),
$$

which means the Rayleigu's friction does not change the structure of classical NavierStokes equation (1.1). Moreover, the regularity also holds.

Remark 2.2. Consider the 3D Navier-Stokes-Voight equation just as [14] with same boundary condition in Lipschitz-like domain

$$
\left\{\begin{array}{l}
\frac{\partial u}{\partial t}-\alpha^{2} \Delta u_{t}-v \Delta u+(u \cdot \nabla) u+\nabla p=f(t, x) \\
\operatorname{div} u=0
\end{array}\right.
$$

then we can not derive the well-posedness for (2.22), since the estimate of background function for the steady state Stokes problem (1.3) is open in three dimension. However, if we consider (2.22) in two dimension Lipschitz domain, then the equation (2.22) has a unique solution $u(t, x)$ satisfying $u(t, x) \in L^{\infty}(\tau, T ; V) \cap L^{2}(\tau, T ; V)$. 


\subsection{The regularity of global weak solution}

By the similar technique in Theorem 2.1 and uniform estimates in $D\left(A^{\frac{\sigma}{2}}\right)$ and $V$, the regularity of global weak solution above can be stated in the following theorem.

Theorem 2.3. (1) Assume $f \in L_{\text {loc }}^{2}(\mathbb{R} ; H)$, let $v(t, x)$ be the solution of (2.20) with the initial data $v_{\tau} \in D\left(A^{\frac{\sigma}{2}}\right)$, then the global weak solution $v(t, x)$ becomes regular as $v(t, x) \in L^{\infty}\left(\tau, T ; D\left(A^{\frac{\sigma}{2}}\right)\right) \cap$ $L^{2}\left(\tau, T ; D\left(A^{\frac{\sigma+1}{2}}\right)\right)$ for $\sigma \in\left[0, \frac{1}{2}\right]$ and $v(t, x) \in C_{w}\left([\tau, T] ; D\left(A^{\frac{\sigma}{2}}\right)\right)$ from the continuous dependence on initial data.

(2) Let $u_{\tau} \in D\left(A^{\frac{\sigma}{2}}\right), f(x, t) \in L_{l o c}^{2}(\mathbb{R} ; H), \varphi \in L^{\infty}(\partial \Omega)$. Then, the problem (1.1) possesses regular solution $u(t, x) \in C_{w}\left([\tau, T] ; D\left(A^{\frac{\sigma}{2}}\right)\right.$.

Proof. See Section 3.2.

Remark 2.3. The recent literature from [15] considered the 3D Navier-Stokes equation with variable viscosity

$$
\left\{\begin{array}{l}
\frac{\partial u}{\partial t}-\left(v+v_{1}\|u\|^{2}\right) \Delta u+(u \cdot \nabla) u+\nabla p=f(t, x), \\
\operatorname{div} u=0 .
\end{array}\right.
$$

If we consider (2.23) with the same condition as (1.1), then the well-posedness and regularity are not easy to achieve, even the two dimension case.

Remark 2.4. In conclusion, from the comparing with some other fluid flow models, we has show that the well-posedness and regularity hold for 2D system since the estimate of background function, even some complex models such as MHD and micropolar flows.

\section{Proof of main results}

In this section, we will prove our main results by some delicate estimates.

\subsection{Proof of Theorems 2.1 and 2.2}

In this section, we shall first prove the global existence of solutions for the equivalent abstract equation of problem (1.1) in $H$. Then the proof of continuous dependence on the initial data and the solution processes will be presented.

\section{- Proof of Theorem 2.1:}

Step 1: We shall use the standard Faedo-Galerkin method to establish the existence of approximate solution to problem (2.20).

Fix $n \geq 1, w_{j}(j \geq 1)$ be the normalized eigenfunctions basis for the Stokes operators in the space $H$ with its increasing eigenvalues satisfying $0<\lambda_{1} \leq \lambda_{2} \leq \cdots$ and $\lim _{j \rightarrow \infty} \lambda_{j}=\infty$. 
Let $V_{n}=\operatorname{span}\left\{w_{1}, w_{2}, \cdots, w_{n}\right\}$, we define an approximate solution $v_{n}$ to problem (2.20) as $v_{n}(t)=\sum_{j=1}^{n} a_{n j}(t) w_{j} \in V_{n}$ which satisfies the following initial value problem of ordinary differential equation with respect to unknown variables $\left\{a_{n j}\right\}_{j=1}^{n}$,

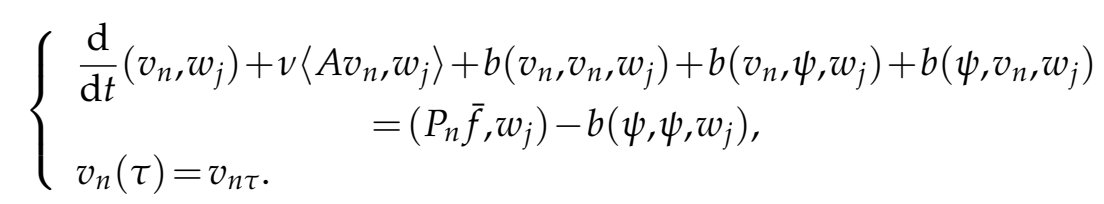

By the local existence theory of solutions for ordinary differential equations, there exists a solution in local interval $(\tau, T)$ for problem (3.1).

Step 2: A priori $L^{\infty}$-estimate.

Multiplying (3.1) by $a_{n j}$, summing the resulting equations from $j=1$ to $n$, noting $b\left(v_{n}, v_{n}, v_{n}\right)=0$ and $b\left(\psi, v_{n}, v_{n}\right)=0$ from (2.3), we have

$$
\frac{1}{2} \frac{\mathrm{d}}{\mathrm{dt}}\left|v_{n}\right|^{2}+v\left\|v_{n}\right\|^{2} \leq\left|b\left(v_{n}, \psi, v_{n}\right)\right|+\left|\left\langle P_{n} \bar{f}, v_{n}\right\rangle\right|+\left|b\left(\psi, \psi, v_{n}\right)\right| .
$$

Next, we shall estimate every term on the right-hand side in (3.2).

(a) Using Hardy's inequality (2.4), (2.3) and (2.11), choosing suitable $\varepsilon>0$ such that

$$
C_{2} C_{3} C_{4} \varepsilon\|\varphi\|_{L^{\infty}(\partial \Omega)} \leq \frac{v}{4}
$$

we obtain

$$
\begin{aligned}
\left|b\left(v_{n}, \psi, v_{n}\right)\right| & \leq C_{4}\|\varphi\|_{L^{\infty}(\partial \Omega)} \int_{\operatorname{dist}(x, \partial \Omega) \leq C_{2} \varepsilon} \operatorname{dist}(x, \partial \Omega) \frac{\left|v_{n}\right|^{2}}{[\operatorname{dist}(x, \partial \Omega)]^{2}} \mathrm{~d} x \\
& \leq C_{2} C_{3} C_{4} \varepsilon\|\varphi\|_{L^{\infty}(\partial \Omega)}\left\|v_{n}\right\|^{2} \leq \frac{v}{4}\left\|v_{n}\right\|^{2} .
\end{aligned}
$$

Similarly, we have

$$
\begin{aligned}
& \left|b\left(\psi, \psi, v_{n}\right)\right| \leq \frac{v}{4}\left\|v_{n}\right\|^{2}+\frac{C \varepsilon|\partial \Omega|}{v}\|\varphi\|_{L^{\infty}(\partial \Omega)^{\prime}}^{2} \\
& \left|<P_{n} \bar{f}, v_{n}>\right| \leq \frac{v}{4}\left\|v_{n}\right\|^{2}+\frac{C}{v}\left[\frac{|f|_{V^{\prime}}^{2}}{\lambda_{1}}+\frac{C v^{2}}{\varepsilon}\|\varphi\|_{L^{2}(\partial \Omega)}^{2}\right] .
\end{aligned}
$$

Combining (3.2)-(3.6), by $\|\varphi\|_{L^{2}(\partial \Omega)}^{2} \leq C|\partial \Omega|\|\varphi\|_{L^{\infty}(\partial \Omega)}^{2}$ and the Poincaré inequality, we get

$$
\begin{aligned}
& \frac{\mathrm{d}}{\mathrm{dt}}\left|v_{n}\right|^{2}+\frac{v \lambda_{1}}{2}\left|v_{n}\right|^{2} \\
\leq & \frac{C}{v}\left[\frac{|f|_{V^{\prime}}^{2}}{\lambda_{1}}+\frac{C v^{2}}{\varepsilon}|\partial \Omega|\|\varphi\|_{L^{\infty}(\partial \Omega)}^{2}+C \varepsilon|\partial \Omega|\|\varphi\|_{L^{\infty}(\partial \Omega)}^{2}\right] \equiv K_{0}^{2} .
\end{aligned}
$$


By the Gronwall inequality, we derive

$$
\left|v_{n}\right|^{2} \leq\left|v_{n \tau}\right|^{2} e^{-\frac{\nu \lambda_{1}}{2}(t-\tau)}+\int_{\tau}^{t} e^{-\frac{\nu \lambda_{1}}{2}(t-s)} K_{0}^{2} \mathrm{~d} s .
$$

Moreover, since $\|\varphi\|_{L^{2}(\partial \Omega)}^{2} \leq C|\partial \Omega|\|\varphi\|_{L^{\infty}(\partial \Omega)}^{2}$ and $f \in L_{l o c}^{2}\left(\tau, T ; V^{\prime}\right)$, then we have, for an arbitrary $t \in(\tau, T)$,

$$
\begin{aligned}
& \int_{\tau}^{t} e^{-\frac{v \lambda_{1}}{2}(t-s)} K_{0}^{2} \mathrm{~d} s \\
= & \int_{\tau}^{t} e^{-\frac{v \lambda_{1}}{2}(t-s)} \frac{C}{v}\left[\frac{|f|_{V^{\prime}}^{2}}{\lambda_{1}}+\frac{v^{2}}{\varepsilon}\|\varphi\|_{L^{2}(\partial \Omega)}^{2}+\varepsilon|\partial \Omega|\|\varphi\|_{L^{\infty}(\partial \Omega)}^{2}\right] \mathrm{d} s \\
= & \int_{\tau}^{t} e^{-\frac{v \lambda_{1}}{2}(t-s)} \frac{C}{v} \frac{|f|_{V^{\prime}}^{2}}{\lambda_{1}} \mathrm{~d} s+\frac{C}{v}\left[\frac{v^{2}}{\varepsilon}|\partial \Omega|\|\varphi\|_{L^{\infty}(\partial \Omega)}^{2}+\varepsilon|\partial \Omega|\|\varphi\|_{L^{\infty}(\partial \Omega)}^{2}\right] \cdot \int_{\tau}^{t} e^{-\frac{v \lambda_{1}}{2}(t-s)} \mathrm{d} s
\end{aligned}
$$

and

$$
\begin{aligned}
& \frac{C}{v}\left[\frac{v^{2}}{\varepsilon}|\partial \Omega|\|\varphi\|_{L^{\infty}(\partial \Omega)}^{2}+\varepsilon|\partial \Omega|\|\varphi\|_{L^{\infty}(\partial \Omega)}^{2}\right] \int_{\tau}^{t} e^{-\frac{v \lambda_{1}}{2}(t-s)} \mathrm{d} s \leq C, \\
& \frac{C}{v \lambda_{1}} \int_{0}^{t} e^{-\frac{\nu \lambda_{1}}{2}(t-s)}|f(s)|_{V^{\prime}}^{2} \mathrm{~d} s \leq \frac{C}{v \lambda_{1}} \int_{\tau}^{t}|f(s)|_{V^{\prime}}^{2} \mathrm{~d} s \leq C .
\end{aligned}
$$

This implies that $v(t, x) \in L^{\infty}(\tau, T ; H)$.

Step 3: The priori $L^{2}$-estimate.

Integrating (3.2) over $(s, t)$, and using (3.3)-(3.6), we have

$$
\begin{aligned}
& \left|v_{n}\right|^{2}+\frac{v}{2} \int_{s}^{t}\left\|v_{n}(r)\right\|^{2} \mathrm{~d} r \\
\leq & \left|v_{n}(s)\right|^{2}+C\|f\|_{L^{2}\left(\tau, T ; V^{\prime}\right)}+\frac{C}{v}\left[\frac{v^{2}}{\varepsilon}|\Omega|\|\varphi\|_{L^{\infty}(\partial \Omega)}^{2}+\varepsilon|\partial \Omega|\|\varphi\|_{L^{\infty}(\partial \Omega)}^{2}\right](t-s),
\end{aligned}
$$

and using the Poincaré inequality and Gronwall's inequality, we derive that

$$
\begin{aligned}
\left|v_{n}\right|^{2} \leq \mid v_{n}(s) & \left.\right|^{2} e^{-\frac{\nu \lambda_{1}}{2}(t-s)}+C\|f\|_{L_{l o c}^{2}\left(\tau, T ; V^{\prime}\right)} e^{-\frac{v \lambda_{1}}{2}(t-s)} \\
+ & \frac{C}{v}\left[\frac{v^{2}}{\varepsilon}|\Omega|\|\varphi\|_{L^{\infty}(\partial \Omega)}^{2}+\varepsilon|\partial \Omega|\|\varphi\|_{L^{\infty}(\partial \Omega)}^{2}\right] \frac{t-s}{e^{\frac{\nu \lambda_{1}}{2}(t-s)}},
\end{aligned}
$$

which shows that $v_{n}(t, x) \in L^{\infty}(\tau, T ; H) \cap L^{2}(\tau, T ; V)$ if let $s=\tau$ in (3.12).

Step 4: The priori $L^{2}$-estimate of $\mathrm{d} v_{n} / \mathrm{d} t$.

Using the estimate of trilinear operator $|b(u, v, w)| \leq C|u|^{\frac{1}{2}}\|u\|^{\frac{1}{2}}\|v\|\|w\|^{1 / 2}|w|^{1 / 2}$ and the Poincaré inequality, we have

$$
\left\|B\left(v_{n}, v_{n}\right)\right\|_{V^{\prime}}=\sup _{\|w\|=1}\left\|b\left(v_{n}, v_{n}, w\right)\right\| \leq C\left|v_{n}\right|^{\frac{1}{2}}\left\|v_{n}\right\|^{\frac{1}{2}}\left\|v_{n}\right\|,
$$




$$
\int_{\tau}^{T}\left\|B\left(v_{n}, v_{n}\right)\right\|_{V^{\prime}}^{2} \mathrm{~d} s \leq C\left(\int_{\tau}^{T}\left|v_{n}\right|^{2} \mathrm{~d} s+\int_{\tau}^{T}\left\|v_{n}\right\|^{2} \mathrm{~d} s\right),
$$

which implies $B\left(v_{n}, v_{n}\right) \in L^{2}\left(\tau, T ; V^{\prime}\right)$.

Considering $\left\langle\frac{\mathrm{d} v_{n}}{\mathrm{~d} t}, w\right\rangle$ with $w \in V$ in the equation

$$
\begin{aligned}
& \left(\frac{\mathrm{d} v_{n}}{\mathrm{~d} t}, w\right)+\left(v A v_{n}, w\right)+\left(B\left(v_{n}, v_{n}\right), w\right)+\left(B\left(v_{n}, \psi\right), w\right)+\left(B\left(\psi, v_{n}\right), w\right) \\
= & (P \bar{f}, w)-(B(\psi, \psi), w),
\end{aligned}
$$

and using the Hardy inequality, Hölder's inequality and the same technique as in (3.4), we deduce

$$
\begin{aligned}
& \mid b\left(v_{n}, \psi, w \mid\right. \leq C_{4}\|\varphi\|_{L^{\infty}(\partial \Omega)} \int_{\operatorname{dist}(x, \partial \Omega) \leq C_{2} \varepsilon} \frac{\left|v_{n}\right|}{\operatorname{dist}(x, \partial \Omega)}|w| \operatorname{dist}(x, \partial \Omega) \mathrm{d} x \\
& \leq C\|\varphi\|_{L^{\infty}(\partial \Omega)}\left\|v_{n}\right\|\|w\|, \\
& \int_{\tau}^{T}\left|B\left(v_{n}, \psi\right)\right|_{V^{\prime}}^{2} \mathrm{~d} s \leq C\|\varphi\|_{L^{\infty}(\partial \Omega)}^{2}\left\|v_{n}\right\|_{L^{2}(\tau, T ; V)}^{2}
\end{aligned}
$$

which means that $B\left(v_{n}, \psi\right) \in L^{2}\left(\tau, T ; V^{\prime}\right)$. Similarly, we have $B\left(\psi, v_{n}\right) \in L^{2}\left(\tau, T ; V^{\prime}\right)$ and $B(\psi, \psi) \in L^{2}\left(\tau, T ; V^{\prime}\right)$. Since $f \in L_{l o c}^{2}\left(\tau, T ; V^{\prime}\right)$ and $v_{n} \in L^{2}(\tau, T ; V)$, then $P \bar{f} \in L^{2}\left(\tau, T ; V^{\prime}\right)$ and $v A v_{n} \in L^{2}\left(\tau, T ; V^{\prime}\right)$. From the equation (3.14) in the weak sense, we have $\frac{\mathrm{d} v_{n}}{\mathrm{~d} t} \in L^{2}\left(\tau, T ; V^{\prime}\right)$ and $\left\{\frac{\mathrm{d} v_{n}}{\mathrm{~d} t}\right\}$ is bounded.

Step 5: Passing to limit and compact argument to achieve the existence of weak solutions.

From Steps 3 and 4, using the Lions-Aubin compact argument and the dominated convergence theorem, we can extract a subsequence (relabeled as $v_{n}$ ) and derive the existence of function $v \in L^{2}(\tau, T ; V) \cap L^{\infty}(\tau, T ; H)$ with $\frac{\mathrm{d} v}{\mathrm{~d} t} \in L^{2}\left(\tau, T ; V^{\prime}\right)$ such that

$$
\begin{array}{ll}
v_{n} \rightarrow v & \text { strongly in } L^{2}(\tau, T ; H), \\
v_{n} \rightarrow v & \text { weakly in } L^{2}(\tau, T ; V), \\
v_{n} \rightarrow v & \text { weakly } \text { in } L^{\infty}(\tau, T ; H), \\
\frac{\mathrm{d} v_{n}}{\mathrm{~d} t} \rightarrow \frac{\mathrm{d} v}{\mathrm{~d} t} & \text { weakly in } L^{2}\left(\tau, T ; V^{\prime}\right) .
\end{array}
$$

Next, we shall deal with the convergence of trilinear operators. Using the Hölder inequality and the property of trilinear operators, we obtain

$$
\begin{aligned}
& \int_{\tau}^{T}\left|b\left(v_{n}, v_{n}, \omega_{j}\right)-b\left(v, v, \omega_{j}\right)\right| \mathrm{d} t \\
= & \int_{\tau}^{T}\left|b\left(v_{n}, v_{n}-v, \omega_{j}\right)\right| \mathrm{d} t \leq C \int_{\tau}^{T}\left\|v_{n}\right\| \| \omega_{j}||\left|v_{n}-v\right| \mathrm{d} t
\end{aligned}
$$




$$
\leq C\left\|v_{n}\right\|_{L^{2}(\tau, T ; V)}\left\|v_{n}-v\right\|_{L^{2}(\tau, T ; H)} \rightarrow 0
$$

Similarly, we have

$$
\begin{aligned}
& \int_{\tau}^{T}\left|b\left(v_{n}-v, v, \omega_{j}\right)\right| \mathrm{d} t \leq \frac{C}{\sqrt{\lambda_{1}}}\|v\|_{L^{2}(\tau, T ; V)}\left\|v_{n}-v\right\|_{L^{2}(\tau, T ; H)} \rightarrow 0, \\
& \int_{\tau}^{T}\left|b\left(v_{n}, \psi, \omega_{j}\right)-b\left(u, \psi, \omega_{j}\right)\right| \mathrm{d} t \leq C\|\varphi\|_{L^{\infty}(\partial \Omega)}\left\|v_{n}-v\right\|_{L^{2}(\tau, T ; H)} \rightarrow 0, \\
& \int_{\tau}^{T}\left|b\left(\psi, v_{n}, \omega_{j}\right)-b\left(\psi, u, \omega_{j}\right)\right| \mathrm{d} t \leq C\|\varphi\|_{L^{\infty}(\partial \Omega)}\left\|v_{n}-v\right\|_{L^{2}(\tau, T ; H)} \rightarrow 0 .
\end{aligned}
$$

Combining (3.17)-(3.24), passing to the limit of (3.14), we conclude that $v(t, x)$ is a weak solution to problem (2.19) in the interval $(\tau, T)$, i.e., there exist at least one global in time Hadamard weak solution to problem (2.19). From the property of background flows class $\psi_{\varepsilon}=\psi \in C^{\infty}(\Omega)$ satisfying (1.3) and $v=u-\psi$ and the solution $v$ for problem (2.19) is obtained in Theorem 2.1 with initial data $v_{\tau}=u_{\tau}-\psi$, it is easy to check that $u$ satisfies the conditions (i), (ii) and (iii) in Definition 3.1 and $u(t, x) \in L^{\infty}(0, T ; H) \cap L^{2}(0, T ; V)$. We then complete the existence result in Theorem 2.1.

\section{- Proof of uniqueness in Theorem 2.1 and Theorem 2.2}

Let $u_{1}(\cdot)$ and $u_{2}(\cdot)$ be two solutions to problem (1.1) with corresponding initial data $u_{\tau}^{1}$ and $u_{\tau}^{2}$ respectively and background flow functions $\psi_{1}$ and $\psi_{2}$, if we take $w=u_{1}-u_{2}$, then $w$ satisfies the problem:

$$
\left\{\begin{array}{l}
\frac{\mathrm{d} w}{\mathrm{~d} t}-v \Delta w+\left(u_{1} \cdot \nabla\right) u_{1}-\left(u_{2} \cdot \nabla\right) u_{2}=0 \\
\operatorname{div} w=0, \quad(x, t) \in \Omega_{\tau} \\
\left.w(t, x)\right|_{\partial \Omega}=0, \quad(x, t) \in \partial \Omega_{\tau} \\
w(\tau, x)=w_{\tau}=u_{\tau}^{1}(x)-u_{\tau}^{2}(x)
\end{array}\right.
$$

which can be written as

$$
\left\{\begin{array}{l}
\frac{\mathrm{d} w}{\mathrm{~d} t}+v A w+B\left(u_{1}, u_{1}\right)-B\left(u_{2}, u_{2}\right)=0 \\
\operatorname{div} w=0
\end{array}\right.
$$

Let $\omega \in C_{0}^{\infty}(\Omega), \operatorname{div} \omega=0$, from the condition (ii) in Definition 2.1, we can derive

$$
\frac{\mathrm{d}}{\mathrm{dt}}<u_{1}-u_{2}, \omega>-v<u_{1}-u_{2}, \Delta \omega>=\int_{\Omega} \sum_{i, j=1}^{2}\left(u_{1}^{i} u_{1}^{j}-u_{2}^{i} u_{2}^{j}\right) \frac{\partial \omega^{i}}{x_{j}} \mathrm{~d} x .
$$

Obviously, (3.27) holds for any $\omega \in V$. In fact, from the condition (ii) and $\left\langle u_{1}-u_{2}, \Delta \omega\right\rangle=$ $-\left(\left(u_{1}-u_{2}, \omega\right)\right)$, we have

$$
u_{1}-u_{2}=\left(u_{1}-\psi_{1}\right)-\left(u_{2}-\psi_{2}\right)+\left(\psi_{1}-\psi_{2}\right) \in L^{2}([0, T] ; V),
$$




$$
\frac{\mathrm{d}}{\mathrm{dt}}\left(u_{1}-u_{2}\right) \in L^{2}\left([0, T] ; V^{\prime}\right), \quad \text { for } \omega \in V \text {. }
$$

Let $\omega=u_{1}-u_{2}$ in (3.27), we have

$$
\begin{aligned}
& \frac{1}{2} \frac{\mathrm{d}}{\mathrm{dt}}|\omega|^{2}+v\|\omega\|^{2} \\
\leq & C \int_{\Omega}\left|u_{2}\right||\omega||\nabla \omega| \mathrm{d} x \leq\left\|u_{2}\right\|_{L^{4}}|\nabla \omega|^{1 / 2}|w|^{1 / 2}|\nabla \omega| \\
\leq & v\|\omega\|^{2}+C_{v}\left\|u_{2}\right\|_{L^{4}}^{4}|\omega|^{2} .
\end{aligned}
$$

Since

$$
\begin{aligned}
\left\|u_{2}\right\|_{L^{4}(\Omega)} & \leq\left\|u_{2}-\psi\right\|_{L^{4}(\Omega)}+\|\psi\|_{L^{4}(\Omega)} \\
& \leq C\left\|\nabla\left(u_{2}-\psi\right)\right\|_{L^{2}(\Omega)}^{\frac{1}{2}}\left\|u_{2}-\psi\right\|_{L^{2}(\Omega)}^{\frac{1}{2}}+\|\psi\|_{L^{4}(\Omega)},
\end{aligned}
$$

we have $u \in L^{4}(\Omega \times(0, T))$ and $\omega(\cdot, 0)=0$, hence $\omega=0$, i.e., the solution is unique. Moreover, we have the continuous dependence on the initial data

$$
\left|u_{1}(s)-u_{2}(s)\right|^{2} \leq\left\|u_{0}^{1}-u_{0}^{2}\right\|_{H}^{2} \times e^{C_{v} \int_{\tau}^{t}\left\|u_{2}(s)\right\|_{L^{4}(\Omega)}^{4} \mathrm{~d} s} .
$$

Note that (3.28) can be written as

$$
\frac{\mathrm{d}}{\mathrm{dt}}|\omega|^{2}+v\|\omega\|^{2} \leq C_{v}^{\prime}\left\|u_{2}\right\|_{L^{4}}^{4}|\omega|^{2}
$$

Integrating from $\tau$ to $t$, we have

$$
|\omega(t)|^{2}+v \int_{\tau}^{t}\|\omega(s)\|^{2} \mathrm{~d} s \leq\left|\omega_{\tau}\right|^{2}+C_{v}^{\prime} \int_{\tau}^{t}\left\|u_{2}(s)\right\|_{L^{4}}^{4}|\omega(s)|^{2} \mathrm{~d} s .
$$

Neglecting the first term on the left-hand side of (3.32), and using (3.30), we derive

$$
\begin{aligned}
& \int_{\tau}^{t}\left\|u_{1}(s)-u_{2}(s)\right\|^{2} \mathrm{~d} s \\
\leq & \frac{1}{v}\left|u_{\tau}^{1}-u_{\tau}^{2}\right|^{2} \times\left(C_{v}^{\prime} \int_{\tau}^{t}\left\|u_{2}(s)\right\|_{L^{4}}^{4} \mathrm{~d} s e^{C_{v} \int_{\tau}^{t}\left\|u_{2}(s)\right\|_{L^{4}(\Omega)}^{4} \mathrm{~d} s}+1\right) .
\end{aligned}
$$

Hence, (3.30) and (3.33) imply the continuous dependent on the initial data for the global weak solutions, and hence $u(t, x) \in C(0, T ; H) \cap L^{2}(0, T ; V)$, which completes the proof. 


\subsection{Proof of Theorem 2.3}

Firstly, we shall present a lemma for the following proof.

Lemma 3.1. For $u \in D\left(A^{1 / 4}\right)$, there exists a constant $C$ such that

$$
\begin{aligned}
& \|u\|_{L^{4}(\Omega)} \leq C\left\|A^{1 / 4} u\right\|_{L^{2}(\Omega)} \\
& \int_{\Omega} \frac{|u(x)|^{2}}{\operatorname{dist}(x, \partial \Omega)} \mathrm{d} x \leq C\left\|A^{1 / 4} u\right\|_{L^{2}(\Omega)}^{2}
\end{aligned}
$$

hold, here $C$ is independent on the domain $\Omega$. Moreover, the following extended estimate

$$
\int_{\Omega} \frac{\left|A^{\alpha} u(x)\right|^{2}}{\operatorname{dist}(x, \partial \Omega)} \mathrm{d} x \leq C\left\|A^{\alpha+1 / 4} u\right\|_{L^{2}(\Omega)}^{2}
$$

is also true.

Proof. See [1].

\section{- Proof of Theorem 2.3: Regularity of global weak solution}

Using the Galerkin approximated technique and compact argument again, noting that $v=u-\psi$, we need to estimate the norms of $v$ in some more regular norm for the nonautonomous problem, i.e., the asymptotic regularity of $v$, the key point of difficulty is the estimate of trilinear operator.

Multiplying the limiting equation with $A^{\sigma} v$ for (2.20), we have

$$
\begin{aligned}
& \quad \frac{1}{2} \frac{\mathrm{d}}{\mathrm{dt}}\left\|A^{\sigma / 2}\right\|^{2}+v\left\|A^{\frac{\sigma+1}{2}} v\right\|^{2} \\
& \leq\left|b\left(v, v, A^{\sigma} u\right)\right|+\left|b\left(v, v, A^{\sigma} u\right)\right|+\left|b\left(\psi, v, A^{\sigma} u\right)\right| \\
& \quad+\left|b\left(v, \psi, A^{\sigma} u\right)\right|+\left|b\left(\psi, \psi, A^{\sigma} u\right)\right|+\left|<\bar{f}, A^{\sigma} v>\right| .
\end{aligned}
$$

Using the same technique in Section 3.1 and Lemma 3.1, we derive that

$$
\begin{aligned}
\left|b\left(v, v, A^{\sigma} v\right)\right| & \leq C\left|A^{1 / 4} v\right|\left|A^{1 / 2}\right|\left|A^{\sigma+\frac{1}{4}} v\right| \leq \frac{v}{8}\left|A^{\frac{\sigma+1}{2}} v\right|^{2}+C\left|A^{1 / 4} v\right|^{2}\left|A^{1 / 2} v\right|^{2} \\
\left|b\left(\psi, v, A^{\sigma} v\right)\right| & \leq C\|\varphi\|_{L^{\infty}(\partial \Omega)}\left|A^{1 / 2}\right|\left|A^{\sigma} v\right| \leq \frac{v}{8}\left|A^{\frac{\sigma+1}{2}} v\right|^{2}+C\|\varphi\|_{L^{\infty}(\partial \Omega)}^{2}\left|A^{1 / 2} v\right|^{2}, \\
\left|b\left(v, \psi, A^{\sigma} v\right)\right| & \leq C\|\varphi\|_{L^{\infty}(\partial \Omega)} \int_{\Omega} \frac{|v(x)|}{\operatorname{dist}(x, \partial \Omega)}\left|A^{\sigma} v\right| \mathrm{d} x \\
& \leq \frac{v}{8}\left|A^{\frac{\sigma+1}{2}} v\right|^{2}+C\|\varphi\|_{L^{\infty}(\partial \Omega)}^{2}\left|A^{1 / 2} v\right|^{2} \\
\left|b\left(\psi, \psi, A^{\sigma} v\right)\right| & \leq C\|\varphi\|_{L^{\infty}(\partial \Omega)}\|\varphi\|_{L^{2}(\partial \Omega)} \int_{\Omega} \frac{\left|A^{\sigma} v(x)\right|}{\operatorname{dist}(x, \partial \Omega)} \mathrm{d} x \\
& \leq \frac{v}{8}\left|A^{\frac{\sigma+1}{2}} v\right|^{2}+C\|\varphi\|_{L^{\infty}(\partial \Omega)}^{2}\|\varphi\|_{L^{2}(\partial \Omega)}^{2} .
\end{aligned}
$$


In addition, by the similar technique in Section 3.1, it yields

$$
\left|<\bar{f}, A^{\sigma} v>\right| \leq\left|<f, A^{\sigma} v>\right|+\left|F, A^{\sigma} v\right| \leq \frac{v}{8}\left|A^{\frac{\sigma+1}{2}} v\right|^{2}+C\left[|f|^{2}+v \sqrt{\varepsilon}|F|^{2}\right] .
$$

Combining (3.37)-(3.42), noting that $f \in L_{l o c}^{2}(\tau, T ; H)$, by the Gronwall inequality, we can conclude the uniformly boundedness of $v$ in $D\left(A^{\frac{\sigma}{2}}\right)$, and hence also $u$, which complete the proof.

\section{Further research}

In this paper, we have given a proof of well-posedness for 2D non-autonomous NS equation in Lipschitz-like domain, our objective next is to study the pullback dynamic systems for our problem, and moreover for the system with delay terms.

\section{Acknowledgement}

This work was partially supported by the Key Project of Science and Technology of Henan Province (Grant No. 182102410069) and The Project of Young Backbone Teacher in Henan Province (No. 2018GGJS039).

\section{References}

[1] Brown R. M., Perry P. A. and Shen Z., On the dimension of the attractor of the nonhomogeneous Navier-Stokes equations in non-smooth domains. Indiana Univ. Math. J. 49 (2000), 81-112.

[2] Fabes E. B., Kenig C. E. and Verchota G. C., The Dirichlet problem for the Stokes system on Lipschitz domains. Duke Math. J. 57 (1988), 769-793.

[3] Foias C., Manley O., Rosa R. and Temam R., Navier-Stokes Equations and Turbulence, Cambridge University Press, Cambridge, 2001.

[4] Hopf E., Üeber die Anfangswertaufgable für die hydrodynamischen Grundgleichungen. Math. Nachr. 4 (1951), 213-231.

[5] Ladyzhenskaya O. A., The Mathematical Theory of Viscous Incompressible Flow, 2nd English edition, Gordon and Breach, New York-London-Paris 1968.

[6] Leray J., Etude de diverses equations integrales nonlineaires et de quelques problemes que pose l'hydrodynamique. J. Math. Pures Appl. 12 (1933), 1-82.

[7] Leray J., Essai sur le mouvement d'un liquide visqueux emplissant l'espace. Acta Math. 63 (1934), 193-248.

[8] Miranville A. and Wang X., Upper bounded on the dimension of the attractor for nonhomogeneous Navier-Stokes equations. Disc. Cont. Dyn. Syst. 2 (1996), 95-110.

[9] Miranville A. and Wang X., Attractors for nonautonomous nonhomogeneous Navier-Stokes equations. Nonlinearity 10 (1997), 1047-1061.

[10] Shen Z., A note on the Dirichlet problem for the Stokes system in Lipschitz domains. Proc. Amer. Math. Soc. 123(3) (1995), 801-811. 
[11] Temam R., Navier-Stokes Equations, Theory and Numerical Analysis, North-Holland Publishing Company, 1979.

[12] $\mathrm{Wu} \mathrm{D}$. and Zhong C. K., The attractors for the nonhomogeneous nonautonomous NavierStokes equations. J. Math. Anal. Appl. 321 (2006), 426-444.

[13] Yang X., Zhao M. and Hou W., Upper Semi-Continuity of Pullback Attractors for the 2D Non-Autonomous Navier-Stokes Equations with Weak Damping. Acta Math. Sci. Ser. A Chin. Ed. 36(4) (2016), 722-739.

[14] Yang X., Feng B., Maier de Souza T. and Wang T., Long-time dynamics for a non-autonomous Navier-Stokes-Voigt equations in Lipschitz domain. Discrete and Continuous Dynamical Systems B 24(1) (2019), 363-386.

[15] Yang X., Feng B., Wang S., Lu Y. and Ma T. F., Pullback dynamics of 3D Navier-Stokes equations with nonlinear viscosity. Nonlinear Analysis: RWA 48 (2019) 337-361. 